\title{
ANALYTICAL SOLUTION OF STEADY-STATE BEHAVIOR OF BERNOULLI PRODUCTION LINE WITH TWO FINITE BUFFERS
}

UDC 629.5.081

Original scientific paper

\begin{abstract}
Summary
Production system engineering is strongly relied on mathematical models of systems under consideration and analytical solution of the problem has an important role in development and validation of more complex numerical tools. Analytical solution of steadystate behavior of serial Bernoulli production line with three machines and two buffers is considered in this paper based on Markov chain approach. Transition matrices in general case, including three machines with different probabilities of failure and arbitrary occupancy of two buffers, are formulated along with equations for performance measures. The developed theory is illustrated using four different serial Bernoulli lines and the obtained results are compared to those determined using semi-analytical approach via aggregation procedure. Finally, the existing discrepancies are analyzed and pointed out for five different levels of buffers occupancy. From general perspective, good agreement has been found for most of the results within the examined space of system states.
\end{abstract}

Key words: $\quad$ Production system engineering; Bernoulli reliability model; Steady-state response; Analytical solution

\section{Introduction}

\subsection{Problem addressed and main results}

Production system engineering (PSE), an emerging scientific and engineering branch, is an important component of production system analysis, design, utilization, monitoring and maintenance with fundamental intention of gaining continuous and additional profit to production companies while minimizing production losses obtained during technological and transportation operations in workshops. It can be successfully applied in a number of industries, such as automotive, chemical, lumber, household appliances, pharmaceutical, shipbuilding, etc. Moreover, its application range is not restricted by the system scale as it can be applied at single machine level or even be generalized to a level of value chains used for purposes of macroeconomic evaluations, [1]. 
Generally, mathematical models used within PSE can be classified in three basic types, i.e. analytical, semi-analytical and numerical. Unfortunately, due to complexity, exact solutions of PSE problems are known only for narrow band of problems, e.g. Bernoulli serial lines with one buffer, [2]. Since such solutions were not sufficient for actual industrial problems semi-analytical models based on the aggregation principle were developed, [1], [37]. Once formulated, analytical and semi-analytical models can be efficiently applied (within given assumptions) at low computational costs in order to determine properties of the considered production system. Such approach is particularly suitable for the design purposes. On the other hand, numerical or simulation models, e.g. discrete event simulation, offer a wide range of abilities and modelling of complex production system relationships while requiring a significant computational costs as compared to the analytical and semi-analytical ones. Therefore, they are suitable for final analyses of production systems, [8] and [9]. Nevertheless, all three model types should be developed and investigated simultaneously in order to ensure reliable benchmarking and evaluation.

In this context, the present paper is focused on analytical modeling of production system and on the exact solution of steady state behavior of serial Bernoulli production line with three machines and two buffers with finite capacities in particular. First, an exact solution of the simplest case, i.e. Bernoulli line with two machines and one buffer is reconsidered, and the obtained solutions are further expanded to Bernoulli line with three machines and two buffers with finite capacity using Markov chain approach, [1] and [10].

In such a way, analytical solution of the steady sate behavior of serial Bernoulli production line with three machines and two finite buffers is developed and presented for the first time. It can be considered as the first step towards analytical solution of the steady state response of serial Bernoulli line with arbitrary number of machines and buffers of arbitrary capacity. In that respect, new transition matrix and performance measures are derived. Application of the developed theory is demonstrated using four different serial Bernoulli lines and the obtained results are compared to those determined using semi-analytical approach via aggregation procedure, [1]. Finally, the existing discrepancies are analyzed and pointed out for five different levels of buffers occupancy. In general terms, the compared results agree very well, except in some special cases that are discussed in more details. The presented validation of the aggregation procedure using analytical solution of the problem is quite important, as to the present it was benchmarked only using extensive simulations, [1].

\subsection{Brief literature review}

The problem of analytical solution of serial Bernoulli production line with two machines and one buffer has been addressed in many research papers, starting with a pioneering work of Sevast'yanov, [2] where an exact solution of the problem is developed using integral equations. However, such approach led to quite complex system in case of line consisting of three machines and two buffers that could not be solved. Therefore, an approximate method was developed, [11], using analysis of internal and boundary system states. Unfortunately, according to [12], the application of the state based method did not result with general expressions in case of more complex production lines. Further attempts to solve complex production lines problems analytically are reported in [12] in more details, while some authors, e.g. [3], point out the inability of analytical investigation of larger production systems, both directly and recursively.

Further research of Bernoulli production lines was focused on development of semianalytical model, its theoretical properties and application within different case studies. Thus an asymptotic analysis technique for modified model of serial production line, also known as the aggregation procedure was developed in [3]. Investigation of a system-theoretic properties of serial production lines in order to enhance the operability of the designed systems was 
performed in [4], while bottlenecks identification, as one of the most important industrial plant indicators, was considered in [5]. Some of the performance measures are reconsidered and reported in [7]. Apart from the steady-state analysis, transient behavior of Bernoulli serial lines based on the second largest transition matrix eigenvalue was studied in [6]. Along with that, analysis of two unreliable batch machines with a finite buffer between them is reported in [13], while recently a discrete-time model and analytical expressions for performance measures of a single buffer line with two capacity levels was developed in [14].

\subsection{Paper outline}

The reminder of the paper is structured as follows: mathematical modelling applied in cases of lines with one and two buffers and derivation of the analytical solution are presented in Section 2. Application of the developed theory is presented in Section 3 using examples of four different serial Bernoulli lines. Finally, discussion, main conclusions and recommendations for further research are given within Sections 4 and 5.

\section{Mathematical modelling}

Consider a serial Bernoulli production line, Fig. 1, where each machine $m_{i}, i=1, \ldots M$ is up with probability $p_{i}$ and down with probability $1-p_{i}$. Buffers $b_{i}, i=1, \ldots M-1$ of capacity $N_{i}$ are placed between two adjacent machines in order to provide storage for parts that are being processed by the production line. Apart from the standard set of assumptions, [1]:

1. the machines have identical cycle time, $T_{C}$, the time axis is slotted and machines begin operation at the beginning of each time slot,

2. the system is blocked before service,

3. the first machine is never starved and the last machine is never blocked,

4. the machine and buffer status is determined at the beginning of each time slot,

5. each machine status is determined independently from the other,

additional one should be introduced, i.e. the production process that is carried out has to satisfy the law of conservation of mass. Therefore, material can enter the process only through $m_{1}$ and exit the process only through $m_{M}$, i.e. by no means can material neither enter or exit the process through buffers. Although this assumption may seem quite straight forward, its introduction clarifies the derivation process.

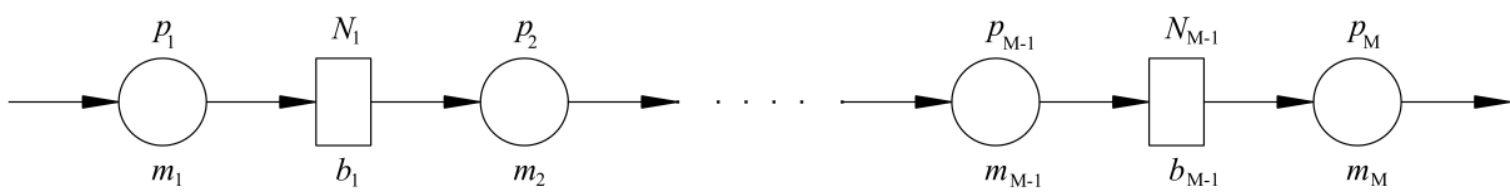

Fig. 1 Serial Bernoulli production line

Given the assumptions, the serial Bernoulli production line can be mathematically formulated as an ergodic Markov chain with the buffer occupancy defining the states of the system. Steady state probabilities of each of these states are determined using balance equations, [15] and [1],

$$
\begin{aligned}
& P_{i}=\sum_{j=1}^{S} P_{i j} P_{j}, i=1,2, \ldots, S, \\
& \sum_{i=1}^{S} P_{i}=1,
\end{aligned}
$$


where $P_{i}$ and $P_{j}$ is steady state probability of state $i$, respectively $j, S$ is the total number of states and $P_{i j}$ is the transition or stochastic matrix. Its important property is that a sum of matrix elements in a column has to be equal to 1 , i.e.

$$
\sum_{i=1}^{S} P_{i j}=1 \text {. }
$$

Moreover, analytic solution of the problem is strongly relied on the stochastic matrix properties, since, according to [15] and [16], in case of homogenous and regular process, its largest eigenvalue is equal to 1 with the associated eigenvector composed of the steady state probabilities, $P_{i}$. Therefore, a system of linear equations (1) can be considered as an eigenvalue problem with unknown eigenvalues and eigenvectors. Once eigenvalues are determined associated eigenvectors can be formulated in a relative, i.e. normalized form. Absolute eigenvectors can easily be obtained afterwards using constraint (2). Such approach is widely used in analysis of the dynamic behavior of systems with two or more degrees of freedom, e.g. [17]. Thus, similarly to vibration analysis, a system (1) has $S$ degrees of freedom, eigenvalues and associated eigenvectors describing "natural response" of the system. Accordingly, the unknown eigenvalues can be determined using expression

$$
\operatorname{det}([P]-\Omega[I])=0 \text {, }
$$

where $[P]$ is transition matrix, $\Omega$ is eigenvalue and $[I]$ unit matrix, while a solution of linear system of equations

$$
\left([P]-\Omega_{k}[I]\right)\{P\}_{k}=\{0\},
$$

gives unknown eigenvectors, $\{P\}_{k}$, normalized to arbitrary vector component and associated to $k$-th eigenvalue, $\Omega_{k}$. Since the analysis of steady state behavior of serial Bernoulli production line is in focus of this paper, only the first eigenvalue, $\Omega_{1}$, and its associated eigenvector, $\{P\}_{1}$, will be considered further on and, due to simplicity, will be denoted without lower indices. In such a way an exact solution can be derived in a simple and straight forward manner. This is first illustrated in a simple case of serial Bernoulli line with two machines and one buffer and extended further on to a more general case.

\subsection{Bernoulli line with two machine and one buffer}

Let us first briefly revisit a serial Bernoulli line with two machines and one buffer, Fig. 2. Machines $m_{1}$, respectively $m_{2}$, are up with probability $p_{1}$, respectively $p_{2}$ and down with probability $1-p_{1}$, respectively $1-p_{2}$. Let us further suppose, for simplicity reasons, that the buffer, $b$, occupancy is equal to $N=2$. Since in case of Bernoulli production line the states of the system are equal to the buffer occupancy, the state space consists of three points as shown at pertaining transition diagram, Fig. 2.

Unknown transition probabilities, $P_{i j}$, can be determined using total probability theorem, conditional probability, [1], defined assumptions and the law of conservation of mass as

$$
[P]=\left[\begin{array}{ccc}
1-p_{1} & \left(1-p_{1}\right) p_{2} & 0 \\
p_{1} & p_{1} p_{2}+\left(1-p_{1}\right)\left(1-p_{2}\right) & \left(1-p_{1}\right) p_{2} \\
0 & p_{1}\left(1-p_{2}\right) & p_{1} p_{2}+\left(1-p_{2}\right)
\end{array}\right]
$$




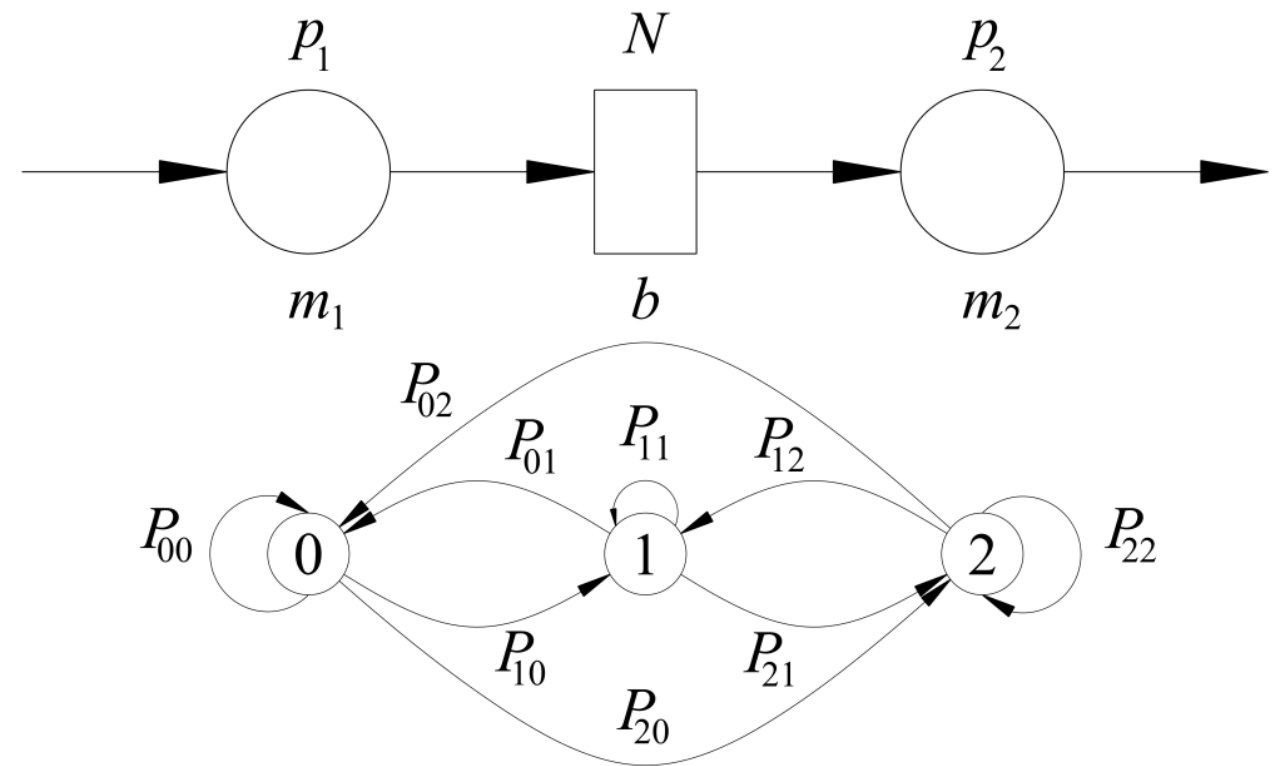

Fig. 2 Serial Bernoulli production line with two machines and one buffer with transition diagram where $[P]$ is transition matrix. Inserting Eq. (6) in Eq. (4) and solving the eigenvalue problem yields the unknown eigenvector

$$
\langle P\rangle=\frac{1}{1-p_{2}+\alpha+\alpha^{2}}\left\langle 1-p_{2}, \alpha, \alpha^{2}\right\rangle,
$$

where

$$
\alpha=\frac{p_{1}\left(1-p_{2}\right)}{p_{2}\left(1-p_{1}\right)} .
$$

In more general case of buffer, $b$, with capacity $N=n$ an eigenvector with $n+1$ components is obtained, [1], i.e.

$$
\langle P\rangle=\frac{1}{1-p_{2}+\alpha+\alpha^{2}+\ldots+\alpha^{n}}\left\langle 1-p_{2}, \alpha, \alpha^{2}, \ldots, \alpha^{n}\right\rangle .
$$

Based on the determined steady state probabilities equations for performance measures involving production rate, $P R$, work-in-process, WIP, probabilities of blockage, $B L$, and starvation, $S T$, can be formulated, [1]. Production rate, $P R$, is defined as the average number of parts processed by the last machine of the production line in steady-state performance. Therefore, in case of one buffer line, it is represented as the intersection of events $\left\{m_{2}\right.$ up $\}$ and $\{b$ not empty $\}$, i.e.

$$
P R=P\left[\left\{m_{2} \text { up }\right\} \cap\{b \text { not empty }\}\right]=p_{2}\left(1-P_{0}\right) .
$$

Average number of parts contained in buffer, $b$, during steady state operation reflects average value of steady-state probabilities and is defined by work-in-process, WIP, that in case of one buffer line takes the form

$$
W I P=\sum_{i=1}^{N} i P_{i},
$$

while probabilities of blockage and starvation, $B L$, respectively, $S T$, represent intersections of events $\left\{m_{1}\right.$ up $\},\{b$ full $\}$ and $\left\{m_{2}\right.$ down $\}$, respectively $\{b$ empty $\}$ and $\left\{m_{2}\right.$ up $\}$, i.e. 


$$
\begin{aligned}
& B L=P\left[\left\{m_{1} \text { up }\right\} \cap\{b \text { full }\} \cap\left\{m_{2} \text { down }\right\}\right]=p_{1} P_{N}\left(1-p_{2}\right), \\
& S T=P\left[\{b \text { empty }\} \cap\left\{m_{2} \text { up }\right\}\right]=P_{0} p_{2} .
\end{aligned}
$$

Detailed derivation procedure in the considered case is presented in [1].

Using the same approach, an exact solution and performance measures of more complex problems involving two buffers with arbitrary capacities can be developed, as presented further on in the paper.

\subsection{Bernoulli line with three machines and two buffers}

Consider a Bernoulli production line with three machines and two buffers, Fig. 3. Machines $m_{1}, m_{2}$ and $m_{3}$ are up with probabilities $p_{1}, p_{2}$ and $p_{3}$, and down with probabilities $1-p_{1}, 1-p_{2}$ and $1-p_{3}$. Similarly to the one buffer case, let us further suppose, that occupancy of buffers $b_{1}$ and $b_{2}$ is equal to $N_{1}=N_{2}=2$. Consequently, according to all possible combinations, the state space consists of nine points as shown at transition diagram, Fig. 3. Since in this case a composition of state space involves double-digit number, transition probabilities involve four indices and are denoted as $P_{i j k l}$ where $i$, respectively $j$, denote occupancy of buffer $b_{1}$, respectively $b_{2}$, in cycle $n+1$ and $k$, respectively $l$, denote occupancy of buffer $b_{1}$, respectively $b_{2}$, in cycle $n$. Due to simplicity, connections between different states are represented at Fig. 3 below and above them, were former represent connections to lower order, and later to higher order states. Self-loops are represented by transition probabilities immediately above states.

Using the same approach as in one buffer line, unknown transition probabilities can be determined based on the defined assumptions using total probability and conditional probability theorems. For clarity purposes, only the case $k=l=1$ is presented, i.e.

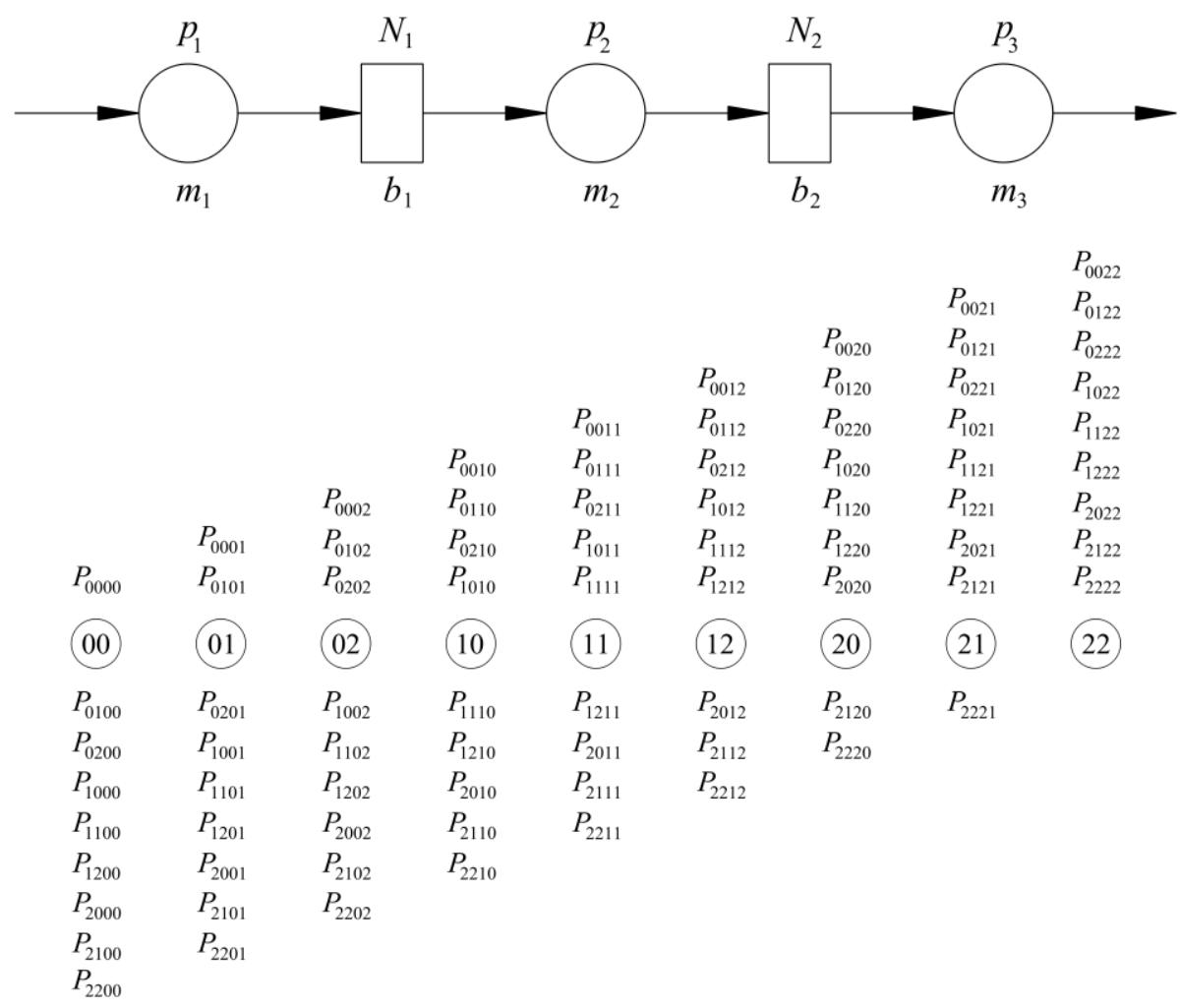

Fig. 3 Serial Bernoulli production line with three machines and two buffers with transition diagram 


$$
\begin{aligned}
& P_{0011}=P\left[h_{1}(n+1)=0, h_{2}(n+1)=0 \mid h_{1}(n)=1, h_{2}(n)=1\right]=0, \\
& P_{0111}=P\left[h_{1}(n+1)=0, h_{2}(n+1)=1 \mid h_{1}(n)=1, h_{2}(n)=1\right]=P\left[\left\{m_{1} \text { down }\right\} \cap\left\{m_{2} \text { up }\right\} \cap\left\{m_{3} \text { up }\right\}\right]=\left(1-p_{1}\right) p_{2} p_{3}, \\
& P_{0211}=P\left[h_{1}(n+1)=0, h_{2}(n+1)=2 \mid h_{1}(n)=1, h_{2}(n)=1\right]=P\left[\left\{m_{1} \text { down }\right\} \cap\left\{m_{2} \text { up }\right\} \cap\left\{m_{3} \text { down }\right\}\right]=\left(1-p_{1}\right) p_{2}\left(1-p_{3}\right), \\
& P_{1011}=P\left[h_{1}(n+1)=1, h_{2}(n+1)=0 \mid h_{1}(n)=1, h_{2}(n)=1\right]=P\left[\left\{m_{1} \text { down }\right\} \cap\left\{m_{2} \text { down }\right\} \cap\left\{m_{3} \text { up }\right\}\right]=\left(1-p_{1}\right)\left(1-p_{2}\right) p_{3}, \\
& P_{1111}=P\left[h_{1}(n+1)=1, h_{2}(n+1)=1 \mid h_{1}(n)=1, h_{2}(n)=1\right]=P\left[\left\{m_{1} \text { up }\right\} \cap\left\{m_{2} \text { up }\right\} \cap\left\{m_{3} \text { up }\right\}\right. \\
& \left.\qquad \cup\left\{m_{1} \text { down }\right\} \cap\left\{m_{2} \operatorname{down}\right\} \cap\left\{m_{3} \operatorname{down}\right\}=p_{1} p_{2} p_{3}+\left(1-p_{1}\right)\left(1-p_{2}\right)\left(1-p_{3}\right)\right], \\
& P_{1211}=P\left[h_{1}(n+1)=1, h_{2}(n+1)=2 \mid h_{1}(n)=1, h_{2}(n)=1\right]=P\left[\left\{m_{1} \text { up }\right\} \cap\left\{m_{2} \text { up }\right\} \cap\left\{m_{3} \text { down }\right\}\right]=p_{1} p_{2}\left(1-p_{3}\right), \\
& P_{2011}=P\left[h_{1}(n+1)=2, h_{2}(n+1)=0 \mid h_{1}(n)=1, h_{2}(n)=1\right]=P\left[\left\{m_{1} \text { up }\right\} \cap\left\{m_{2} \operatorname{down}\right\} \cap\left\{m_{3} \text { up }\right\}\right]=p_{1}\left(1-p_{2}\right) p_{3}, \\
& P_{2111}=P\left[h_{1}(n+1)=2, h_{2}(n+1)=1 \mid h_{1}(n)=1, h_{2}(n)=1\right]=P\left[\left\{m_{1} \text { up }\right\} \cap\left\{m_{2} \operatorname{down}\right\} \cap\left\{m_{3} \text { down }\right\}\right]=p_{1}\left(1-p_{2}\right)\left(1-p_{3}\right), \\
& P_{2211}=P\left[h_{1}(n+1)=2, h_{2}(n+1)=2 \mid h_{1}(n)=1, h_{2}(n)=1\right]=0,
\end{aligned}
$$

where $h_{1}(n)$ and $h_{2}(n)$ represent the state of the first and the second buffer in the $n$-th cycle. Once formulated, transient probabilities can be presented in a form of transition matrix $[P]$

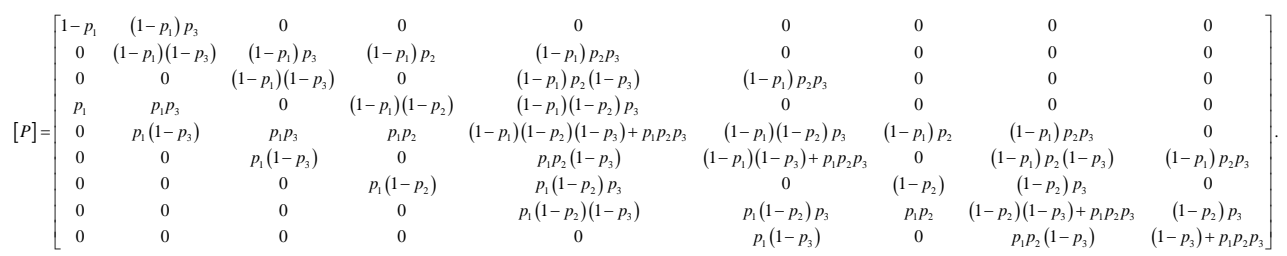

In case of equal probabilities for each machine, i.e. $p_{1}=p_{2}=p_{3}$, Eq. (14) is equal to the one presented by [6].

In general case when $p_{1} \neq p_{2} \neq p_{3}$ and when capacity of buffers is arbitrary integer number, transition matrix elements, $P_{i j k l}$, take the form depending on combination of indexes $i, j, k$ and $l$. Therefore, in case when $k=0$ and $l=0$

$$
P_{i j k l}=\left\{\begin{array}{l}
1-p_{1} \text { for } i=k, j=l, \\
p_{1} \text { for } i=k+1, j=l,
\end{array}\right.
$$

for $k=0$ and $l=1,2, \ldots N_{2}$

$$
P_{i j k l}=\left\{\begin{array}{c}
\left(1-p_{1}\right) p_{3} \text { for } i=k, j=l-1, \\
\left(1-p_{1}\right)\left(1-p_{3}\right) \text { for } i=k, j=l, \\
p_{1} p_{3} \text { for } i=k+1, j=l-1, \\
p_{1}\left(1-p_{3}\right) \text { for } i=k+1, j=l,
\end{array}\right.
$$

and for $k=1,2, \ldots N_{1}-1$ and $l=0$

$$
P_{i j k l}=\left\{\begin{array}{c}
\left(1-p_{1}\right) p_{2} \text { for } i=k-1, j=l+1, \\
\left(1-p_{1}\right)\left(1-p_{2}\right) \text { for } i=k, j=l, \\
p_{1} p_{2} \text { for } i=k, j=l+1, \\
p_{1}\left(1-p_{2}\right) \text { for } i=k+1, j=l .
\end{array}\right.
$$

The internal states, i.e. when $k=1,2, \ldots N_{1}-1$ and $l=1,2, \ldots N_{2}-1$ are defined as 


$$
P_{i j k l}=\left\{\begin{array}{c}
\left(1-p_{1}\right) p_{2} p_{3} \text { for } i=k-1, j=l, \\
\left(1-p_{1}\right) p_{2}\left(1-p_{3}\right) \text { for } i=k-1, j=l+1, \\
\left(1-p_{1}\right)\left(1-p_{2}\right) p_{3} \text { for } i=k, j=l-1, \\
\left(1-p_{1}\right)\left(1-p_{2}\right)\left(1-p_{3}\right)+p_{1} p_{2} p_{3} \text { for } i=k, j=l, \\
p_{1} p_{2}\left(1-p_{3}\right) \text { for } i=k, j=l+1, \\
p_{1}\left(1-p_{2}\right) p_{3} \text { for } i=k+1, j=l-1, \\
p_{1}\left(1-p_{2}\right)\left(1-p_{3}\right) \text { for } i=k+1, j=l .
\end{array}\right.
$$

Finally, the remaining boundary states are equal to: for $k=1,2, \ldots N_{1}-1$ and $l=N_{2}$

$$
P_{i j k l}=\left\{\begin{array}{c}
\left(1-p_{1}\right) p_{2} p_{3} \text { for } i=k-1, j=l, \\
\left(1-p_{1}\right)\left(1-p_{2}\right) p_{3} \text { for } i=k, j=l-1, \\
\left(1-p_{1}\right)\left(1-p_{3}\right)+p_{1} p_{2} p_{3} \text { for } i=k, j=l, \\
p_{1}\left(1-p_{2}\right) p_{3} \text { for } i=k+1, j=l-1 \\
p_{1}\left(1-p_{3}\right) \text { for } i=k+1, j=l,
\end{array}\right.
$$

for $k=N_{1}$ and $l=0$

$$
P_{i j k l}=\left\{\begin{array}{c}
\left(1-p_{1}\right) p_{2} \text { for } i=k-1, j=l+1, \\
1-p_{2} \text { for } i=k, j=l+1, \\
p_{1} p_{2} \text { for } i=k, j=l+1,
\end{array}\right.
$$

for $k=N_{1}$ and $l=1,2, \ldots N_{2}-1$

$$
P_{i j k l}=\left\{\begin{array}{c}
\left(1-p_{1}\right) p_{2} p_{3} \text { for } i=k-1, j=l, \\
\left(1-p_{1}\right) p_{2}\left(1-p_{3}\right) \text { for } i=k-1, j=l+1, \\
\left(1-p_{2}\right) p_{3} \text { for } i=k, j=l-1, \\
\left(1-p_{2}\right)\left(1-p_{3}\right)+p_{1} p_{2} p_{3} \text { for } i=k, j=l \\
p_{1} p_{2}\left(1-p_{3}\right) \text { for } i=k, j=l+1,
\end{array}\right.
$$

and for $k=N_{1}$ and $l=N_{2}$

$$
P_{i j k l}=\left\{\begin{array}{c}
\left(1-p_{1}\right) p_{2} p_{3} \text { for } i=k-1, j=l, \\
\left(1-p_{2}\right) p_{3} \text { for } i=k, j=l-1, \\
\left(1-p_{3}\right)+p_{1} p_{2} p_{3} \text { for } i=k, j=l .
\end{array}\right.
$$

Inserting Eq. (14) into Eq. (4) and solving the eigenvalue problem gives a set of eigenvalues with the first one equal to unit value, i.e. $\Omega=1$. Therefore, a set of equations can be formulated using Eq. (5) for $\Omega=1$ and solved in terms of $P_{00}$. After some algebraic manipulations solution of the linear system of equations gives the unknown eigenvector $\langle P\rangle=\left\langle P_{00}, P_{01}, P_{02}, P_{10}, P_{11}, P_{12}, P_{20}, P_{21}, P_{22}\right\rangle$ in normalized form. Since in general case, when $p_{1} \neq p_{2} \neq p_{3}$, the resulting eigenvector takes quite bearish form, only two eigenvectors in case of equal and symmetric probability distribution are presented. In the former case, when $p_{1}=p_{2}=p_{3}=p$, the eigenvector $\{P\}$ reads 
where

$$
\begin{aligned}
& \alpha=\frac{1}{1-p}, \\
& \beta=\frac{1}{(2-p)^{2}} .
\end{aligned}
$$

In the latter case the probabilities are distributed symmetrically, i.e. $p_{1}=p_{3}=p_{1}, p_{2} \neq p_{1}$, and the resulting eigenvector takes more complex form

$$
\langle P\rangle=\left\{\begin{array}{l}
P_{00} \\
P_{01} \\
P_{02} \\
P_{10} \\
P_{11} \\
P_{12} \\
P_{20} \\
P_{21} \\
P_{22}
\end{array}\right\}=P_{00}\left\{\begin{array}{c}
1 \\
\alpha \\
\beta \Phi_{02}\left(p_{1}, p_{2}\right) \\
\alpha \beta \Phi_{10}\left(p_{1}, p_{2}\right) \\
\alpha^{2} \beta \gamma \Phi_{11}\left(p_{1}, p_{2}\right) \\
\alpha \beta \Phi_{12}\left(p_{1}, p_{2}\right) \\
\alpha^{3} \beta \gamma^{2} \Phi_{20}\left(p_{1}, p_{2}\right) \\
\alpha^{3} \beta \gamma \Phi_{21}\left(p_{1}, p_{2}\right) \\
\alpha^{2}
\end{array}\right\},
$$

where

$$
\begin{aligned}
& \alpha=\frac{1}{1-p_{1}}, \beta=\frac{1}{-p_{1}^{3} p_{2}+p_{1}^{3}+2 p_{1}^{2} p_{2}-3 p_{1}^{2}-3 p_{1} p_{2}+3 p_{1}+p_{2}}, \gamma=\frac{p_{1}}{p_{2}}, \\
& \Phi_{02}\left(p_{1}, p_{2}\right)=\left(p_{1}^{2} p_{2}-p_{1}^{2}-p_{1} p_{2}+2 p_{1}+p_{2}\right), \\
& \Phi_{10}\left(p_{1}, p_{2}\right)=\left(-p_{1}^{2} p_{2}^{2}-p_{1}^{3} p_{2}+p_{1}^{3}+4 p_{1}^{2} p_{2}+p_{1} p_{2}^{2}-4 p_{1}^{2}-5 p_{1} p_{2}+4 p_{1}+p_{2}\right), \\
& \Phi_{11}\left(p_{1}, p_{2}\right)=\left(p_{1}^{2} p_{2}^{2}+p_{1}^{3} p_{2}-p_{1}^{3}-4 p_{1}^{2} p_{2}-2 p_{1} p_{2}^{2}+4 p_{1}^{2}+6 p_{1} p_{2}-4 p_{1}-2 p_{2}\right), \\
& \Phi_{12}\left(p_{1}, p_{2}\right)=\left(p_{1}^{2} p_{2}-p_{1}^{2}-2 p_{1} p_{2}+3 p_{1}+p_{2}\right), \\
& \Phi_{20}\left(p_{1}, p_{2}\right)=\left(p_{1}^{4} p_{2}+p_{1}^{3} p_{2}^{2}-p_{1}^{4}-6 p_{1}^{3} p_{2}-2 p_{1}^{2} p_{2}^{2}+6 p_{1}^{3}+13 p_{1}^{2} p_{2}+3 p_{1} p_{2}^{2}-12 p_{1}^{2}-p_{2}^{2}-12 p_{1} p_{2}+8 p_{1}+2 p_{2}\right), \\
& \Phi_{21}\left(p_{1}, p_{2}\right)=\left(-p_{1}^{3} p_{2}+3 p_{1}^{3}+2 p_{1}^{2} p_{2}-4 p_{1}^{2}-3 p_{1} p_{2}+4 p_{1}+p_{2}\right) .
\end{aligned}
$$

It can easily be checked that Eqs. (25) and (26) are reduced to Eq. (23) if $p_{1}=p_{2}=p$. As mentioned earlier, symbolic representation of analytic expressions in general case of 
$p_{1} \neq p_{2} \neq p_{3}$ and arbitrary buffer capacity is rather cumbersome and will be considered further in the paper in numerical form.

Based on the determined steady state probabilities, equations for performance measures can be formulated in the similar way as in one buffer case. In case of two buffer line, production rate, $P R$, is defined as the intersection of events $\left\{m_{3} u p\right\}$ and $\left\{b_{2}\right.$ not empty $\}$, i.e.

$$
P R=P\left[\left\{m_{3} \text { up }\right\} \cap\left\{b_{2} \text { not empty }\right\}\right]=p_{3}\left(1-\sum_{k=0}^{N_{1}} P_{k 0}\right) .
$$

Furthermore, a formulation of work-in-process, WIP, has to be provided for each buffer separately as well as cumulative definition valid for the entire line. Therefore, in case of the first and the second buffer, work-in-process, $W I P_{1}$, respectively $W I P_{2}$, are defined as

$$
\begin{aligned}
& W I P_{1}=\sum_{l=0}^{N_{2}} \sum_{k=1}^{N_{1}} k P_{k l}, \\
& W I P_{2}=\sum_{k=0}^{N_{1}} \sum_{l=1}^{N_{2}} l P_{k l},
\end{aligned}
$$

and the resulting work-in-process, WIP, is equal to the sum

$$
W I P=W I P_{1}+W I P_{2} .
$$

Finally, probabilities of blockage $B L_{1}$ and $B L_{2}$ for machines $m_{1}$ and $m_{2}$ as well as probabilities of starvation $S T_{2}$ and $S T_{3}$ for machines $m_{2}$ and $m_{3}$ have to be determined. They are resented as intersections of different combinations of events, i.e.

$$
\begin{gathered}
B L_{1}=P\left[\left\{m_{1} \text { up }\right\} \cap\left\{b_{1} \text { full }\right\} \cap\left\{m_{2} \text { down }\right\} \cup\left\{m_{1} \text { up }\right\}\right. \\
\left.\cap\left\{b_{1} \text { full }\right\} \cap\left\{m_{2} \text { up }\right\} \cap\left\{b_{2} \text { full }\right\} \cap\left\{m_{3} \text { down }\right\}\right]= \\
p_{1} \sum_{l=0}^{N_{2}} P_{N_{1} l}\left(1-p_{2}\right)+p_{1} \sum_{l=0}^{N_{2}} P_{N_{1} l} B L_{2}, \\
B L_{2}=P\left[\left\{m_{2} \text { up }\right\} \cap\left\{b_{2} \text { full }\right\} \cap\left\{m_{3} \text { down }\right\}\right]=p_{2} \sum_{k=0}^{N_{1}} P_{k N_{2}}\left(1-p_{3}\right), \\
S T_{2}=P\left[\left\{b_{1} \text { empty }\right\} \cap\left\{m_{2} \text { up }\right\}\right]=\sum_{l=0}^{N_{2}} P_{0 l} p_{2}, \\
S T_{3}=P\left[\left\{b_{2} \text { empty }\right\} \cap\left\{m_{3} \text { up }\right\}\right]=\sum_{k=0}^{N_{1}} P_{k 0} p_{3} .
\end{gathered}
$$

In such a way, application of Eqs. (13) - (30) results with an exact solution of the steady-state performance measures in case of production line with three machines and two buffers of arbitrary occupancies.

\section{Numerical examples}

Application of the developed analytical solution is demonstrated in cases of four production lines with three machines and two buffers. In the first case line $L_{1}$ is composed of three identical machines with equal probabilities $p_{1}=p_{2}=p_{3}=0.8$ and equal buffer capacities $N_{1}=N_{2}=2$. Line $L_{2}$ is composed of three machines with symmetric distribution of probabilities $p_{1}=p_{3}=0.8, p_{2}=0.9$ and equal buffer capacities $N_{1}=N_{2}=2$, while nonidentical machines with probabilities $p_{1}=0.8, p_{2}=0.9, p_{3}=0.7$ and buffers with capacities $N_{1}=N_{2}=2$ are placed within line $L_{3}$. Line $L_{4}$ is a reverse of line $L_{3}$ with non-identical 
machines and probabilities of failure $p_{1}=0.7, p_{2}=0.9, p_{3}=0.8$. A summary of the lines composition is given in Table 1.

Table 1 Properties of lines $L_{1}, L_{2}, L_{3}$ and $L_{4}$

\begin{tabular}{l|ccccc} 
Line & $p_{1}$ & $p_{2}$ & $p_{3}$ & $N_{1}$ & $N_{2}$ \\
\hline$L_{1}$ & 0.8 & 0.8 & 0.8 & 2 & 2 \\
$L_{2}$ & 0.8 & 0.9 & 0.8 & 2 & 2 \\
$L_{3}$ & 0.8 & 0.9 & 0.7 & 2 & 2 \\
$L_{4}$ & 0.7 & 0.9 & 0.8 & 2 & 2
\end{tabular}

Steady state probabilities determined in cases of lines $L_{1}, L_{2}, L_{3}$ and $L_{4}$ are presented in form of probability mass functions, Figs. 4-5, similarly to spectral decomposition of periodic functions commonly used in vibration analysis, [17]. Based on steady state probabilities performance measures, i.e. production rate, work-in-process and probabilities of blockage and starvation can be calculated using Eqs. (27) - (30). The obtained results are compared to those determined using the aggregation procedure, [1], using PSEToolbox software, [18]. Aggregation procedure consists of backward and forward iterations with cumulative calculation of the line performance measures resulting with an approximate solution of the problem in case of lines with two or more buffers. The results obtained using the developed analytical solution (AS) and aggregation procedure (AP) are compared in Tables 2 and 3 in case of lines $L_{1}, L_{2}, L_{3}$ and $L_{4}$, where relative error is defined as

$$
\varepsilon=\frac{\mathrm{AP}-\mathrm{AS}}{\mathrm{AS}} \cdot 100 \% \text {. }
$$

Table 2 Comparison of production rate and work in process determined using analytic and approximate procedure

\begin{tabular}{l|lll|lll|lll|lll}
\multirow{2}{*}{ Line } & $P R$ & & & $W I P_{1}$ & & & & & & & & \\
& AS & AP & $\varepsilon, \%$ & AS & AP & $\varepsilon, \%$ & AS & AP & $\varepsilon, \%$ & AS & AP & $\varepsilon, \%$ \\
\hline$L_{1}$ & 0.6888 & 0.6888 & 0.00 & 1.481 & 1.47 & -0.74 & 1.232 & 1.22 & -0.97 & 2.713 & 2.69 & -0.85 \\
$L_{2}$ & 0.7293 & 0.7356 & 0.86 & 1.344 & 1.34 & -0.30 & 1.419 & 1.40 & -1.34 & 2.763 & 2.74 & -0.83 \\
$L_{3}$ & 0.6670 & 0.6727 & 0.85 & 1.491 & 1.50 & 0.63 & 1.627 & 1.63 & 0.18 & 3.118 & 3.13 & 0.38 \\
$L_{4}$ & 0.6622 & 0.6727 & 1.58 & 1.089 & 1.05 & -2.15 & 1.228 & 1.17 & -4.72 & 2.317 & 2.22 & -4.18
\end{tabular}

Table 3 Comparison of probabilities of blockage and starvation determined using analytic and approximate procedure

\begin{tabular}{l|lll|lll|lll|lll}
\multirow{2}{*}{ Line } & $B L_{1}$ & & & $B L_{2}$ & & & $S T_{2}$ & & & & & \\
& AS & AP & $\varepsilon, \%$ & AS & AP & $\varepsilon, \%$ & AS & AP & $\varepsilon, \%$ & AS & AP & $\varepsilon, \%$ \\
\hline$L_{1}$ & 0.1125 & 0.1112 & -1.15 & 0.0580 & 0.0577 & -0.65 & 0.0576 & 0.0577 & 0.17 & 0.1112 & 0.1112 & 0.00 \\
$L_{2}$ & 0.0652 & 0.0644 & -1.23 & 0.0891 & 0.0864 & -3.03 & 0.0889 & 0.0864 & -2.81 & 0.0702 & 0.0644 & -8.26 \\
$L_{3}$ & 0.1247 & 0.1273 & 2.08 & 0.1806 & 0.1799 & -0.39 & 0.0628 & 0.0592 & -5.73 & 0.0328 & 0.0273 & -16.77 \\
$L_{4}$ & 0.0298 & 0.0273 & -8.34 & 0.0651 & 0.0592 & -9.06 & 0.1863 & 0.1799 & -3.43 & 0.1376 & 0.1273 & -7.48
\end{tabular}



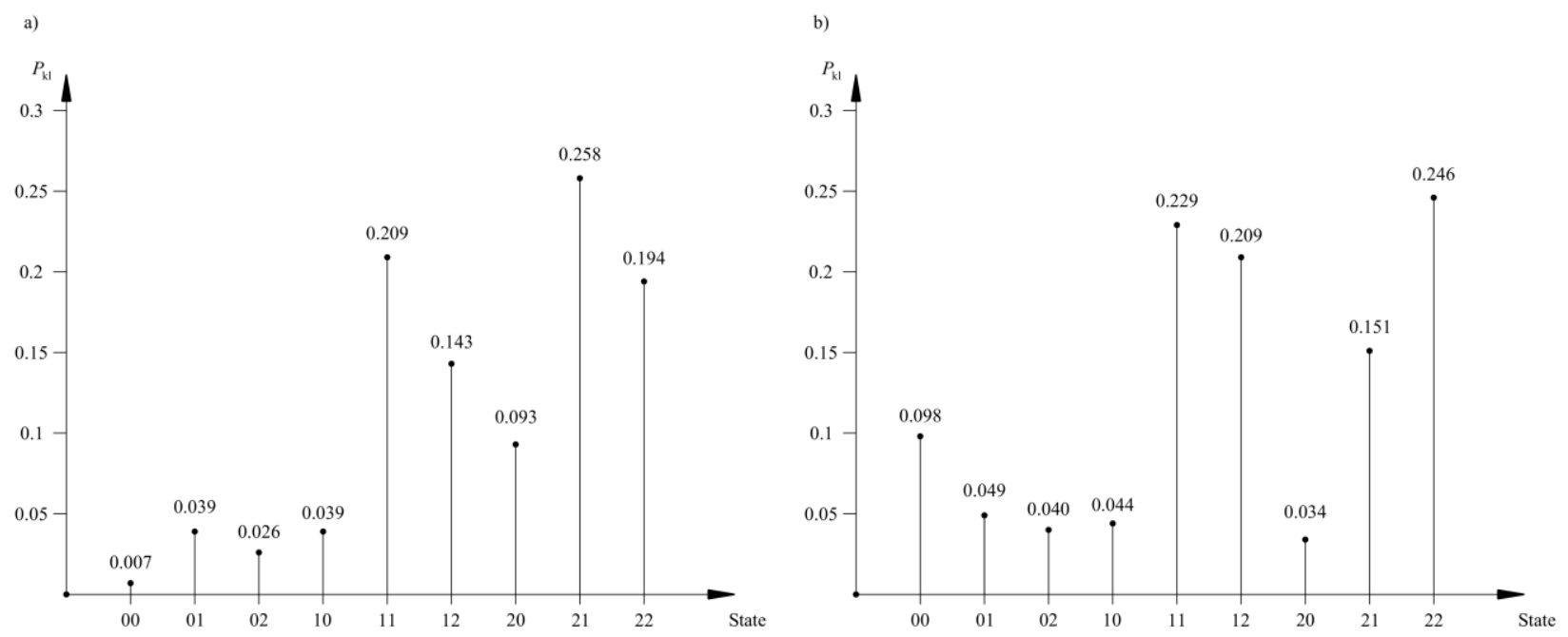

Fig. 4 Stationary probability mass function in case of lines (a) $L_{1}$ and (b) $L_{2}$
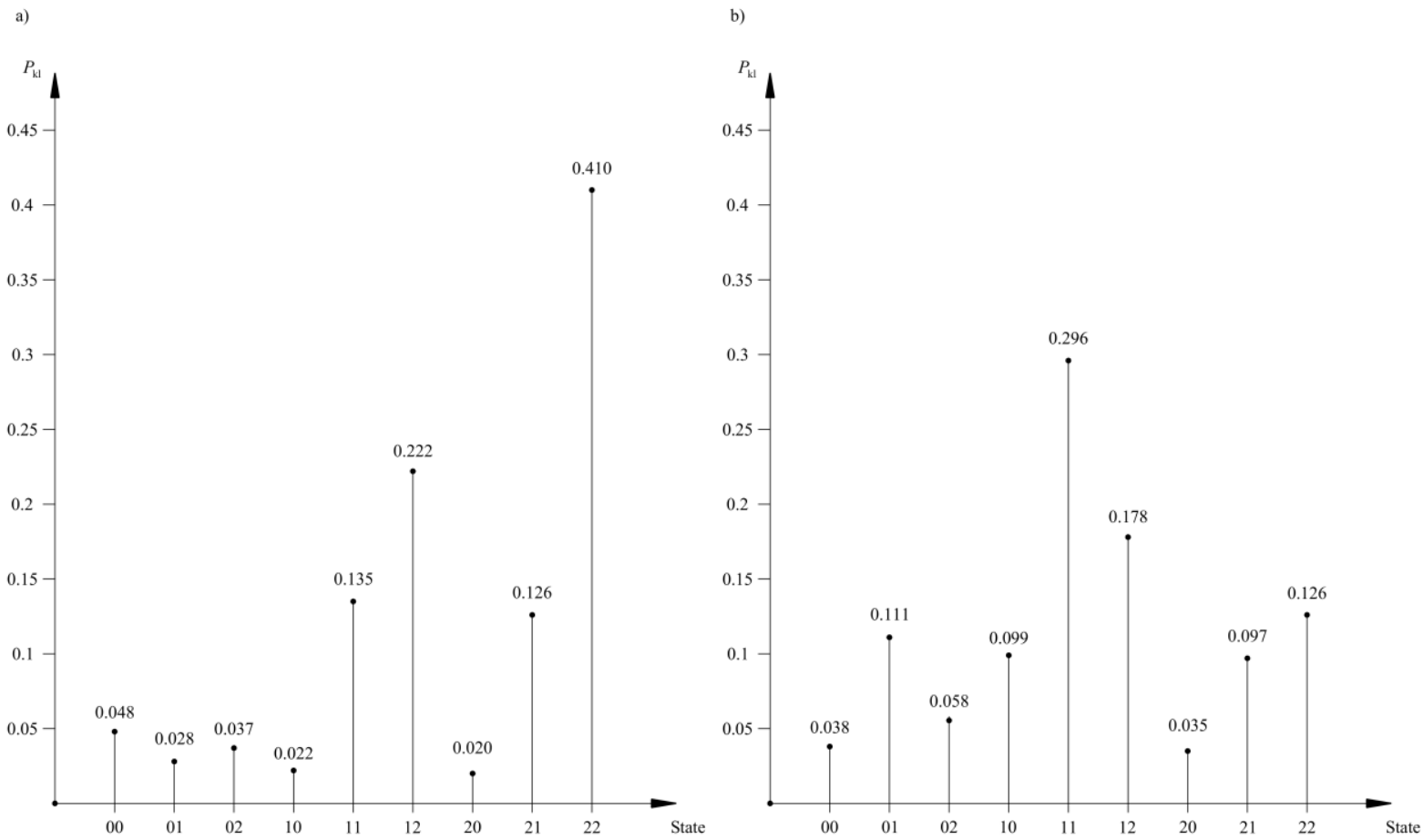

Fig. 5 Stationary probability mass function in case of lines (a) $L_{3}$ and (b) $L_{4}$

A comparison of analytical solution (AS) with results obtained using aggregation procedure (AP) shows that in some cases performance measures are estimated within acceptable limits applicable for engineering purposes, particularly in case of line $L_{1}$ with identical machines. However, significant discrepancies can be noticed in cases of lines with non-identical machines, especially for estimates of probabilities of blockage and starvation where absolute value of relative error reaches up to $16.77 \%$ in case of line $L_{3}$. A reason for such large discrepancies is related to the aggregation procedure algorithm which is based on the equality of production rate of virtual two machine line and the probability of failure of the aggregated machine, [1]. In such a way, due to loss of generality, aggregation procedure does not take into account all of the steady state probabilities influencing performance measures which in turn results with lower accuracy of the estimates, especially in cases of lines 
composed of non-identical machines. This issue could jeopardize aggregation procedure based bottleneck analysis, as it is based on probabilities of blockage and starvation, [1].

Apart from probabilities of failure, an important parameter influencing the complexity of the problem and accuracy of the results is buffer capacity. In order to investigate influence of capacities of buffers $b_{1}$ and $b_{2}$ on results of the analysis, a series of calculations for all considered lines was performed using the same approach, i.e. applying the analytical solution (AS) and the aggregation procedure (AP) for capacities between 1 and 5. A comparison of the results is shown at Figures 6-12.
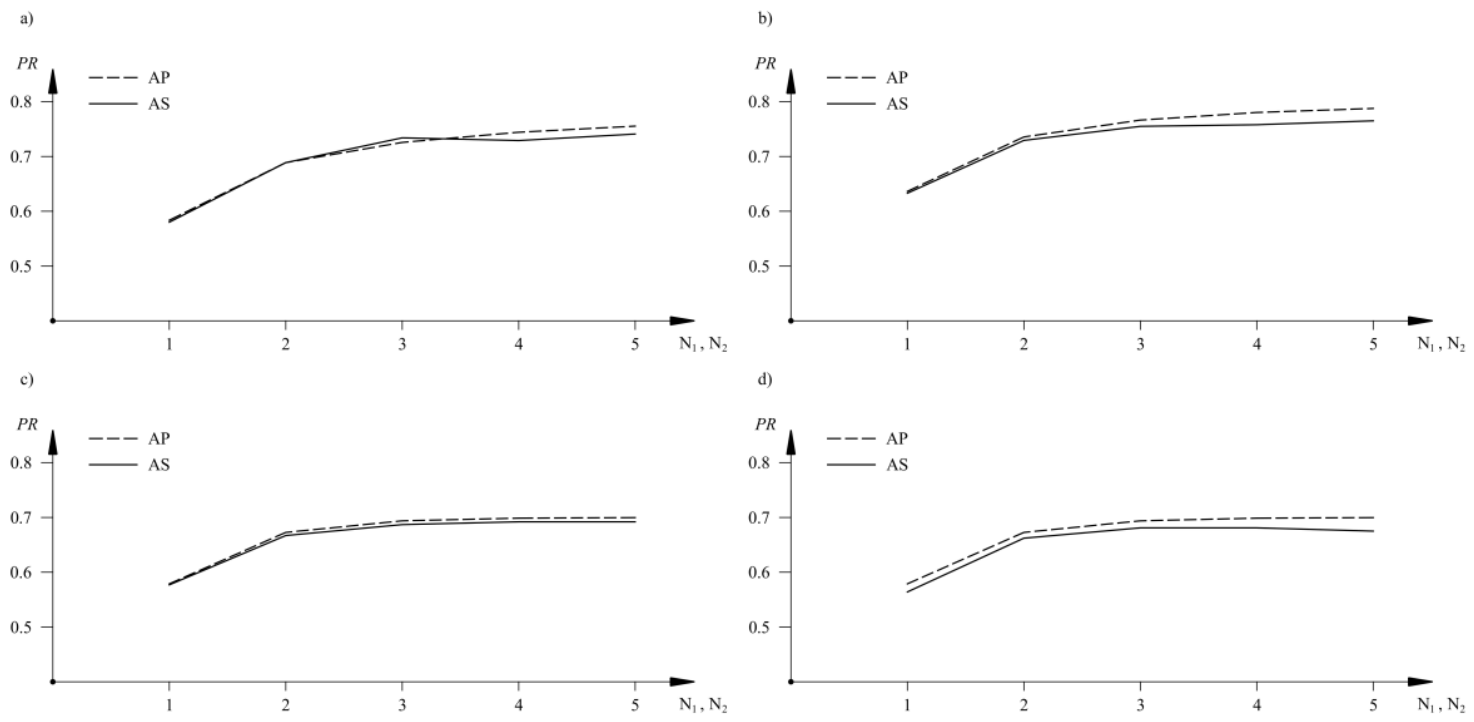

Fig. 6 Influence of capacity of buffers on production rate for line a) $\left.L_{1}, b\right) L_{2}$, c) $L_{3}$, d) $L_{4}$
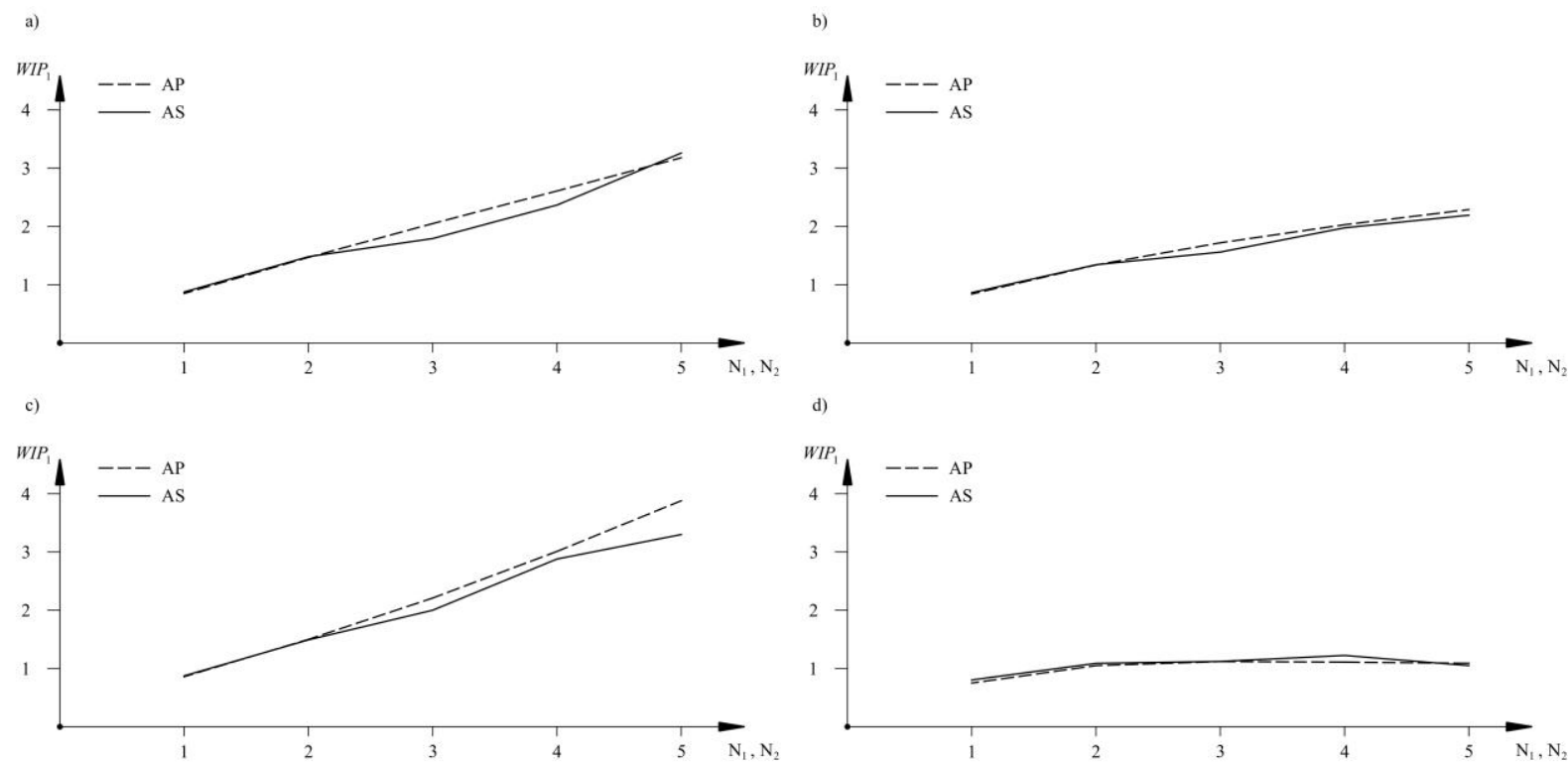

Fig. 7 Influence of capacity of buffers on the $1^{\text {st }}$ machine work-in-process for line a) $L_{1}$, b) $L_{2}$, c) $L_{3}$, d) $L_{4}$ 

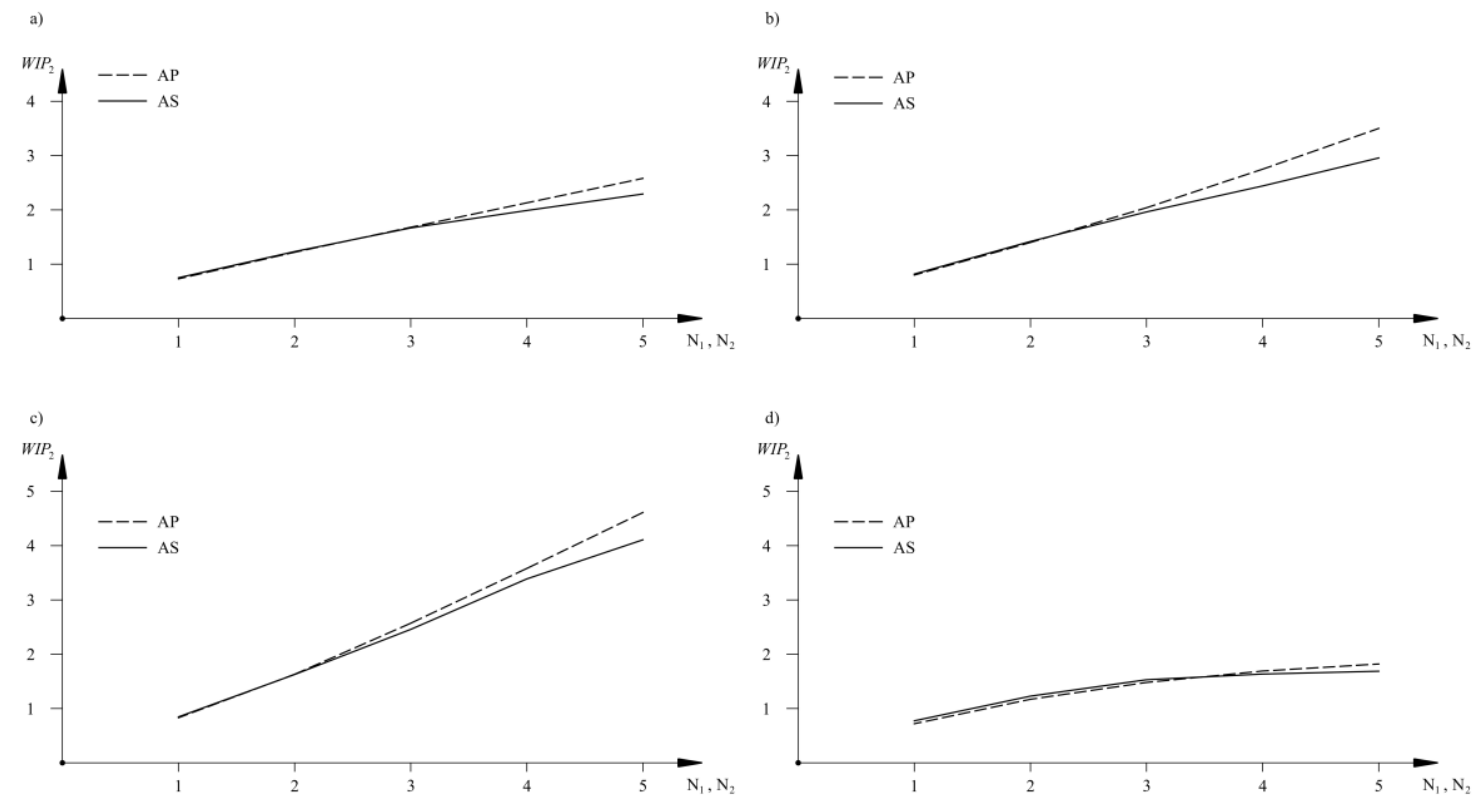

Fig. 8 Influence of capacity of buffers on the $2^{\text {nd }}$ machine work-in-process for line a) $\left.L_{1}, b\right) L_{2}$, c) $L_{3}$, d) $L_{4}$
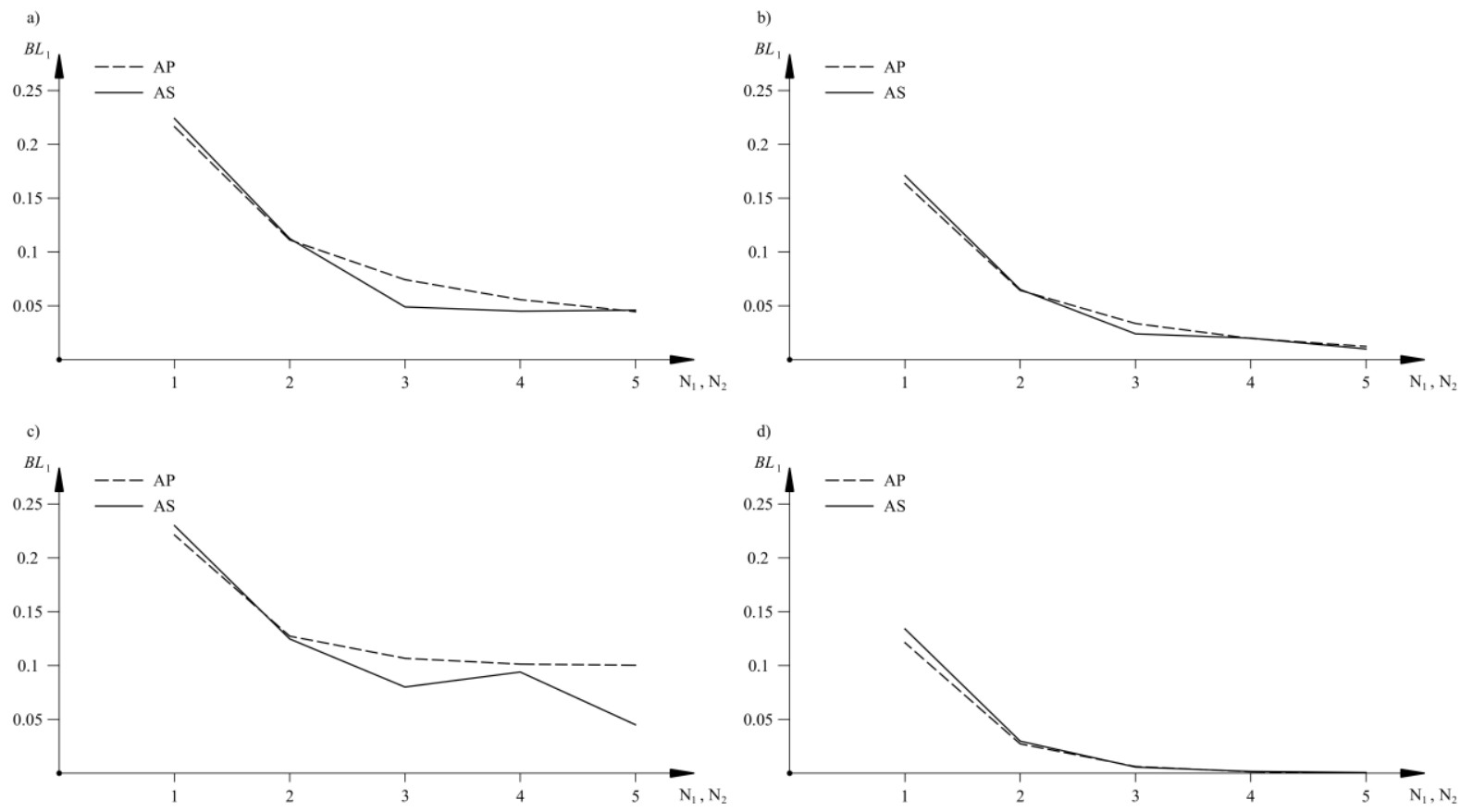

Fig. 9 Influence of capacity of buffers on the probability of blockage of the $1^{\text {st }}$ machine for line a) $L_{1}$, b) $L_{2}$, c) $\mathrm{L}_{3}$, d) $\mathrm{L}_{4}$ 

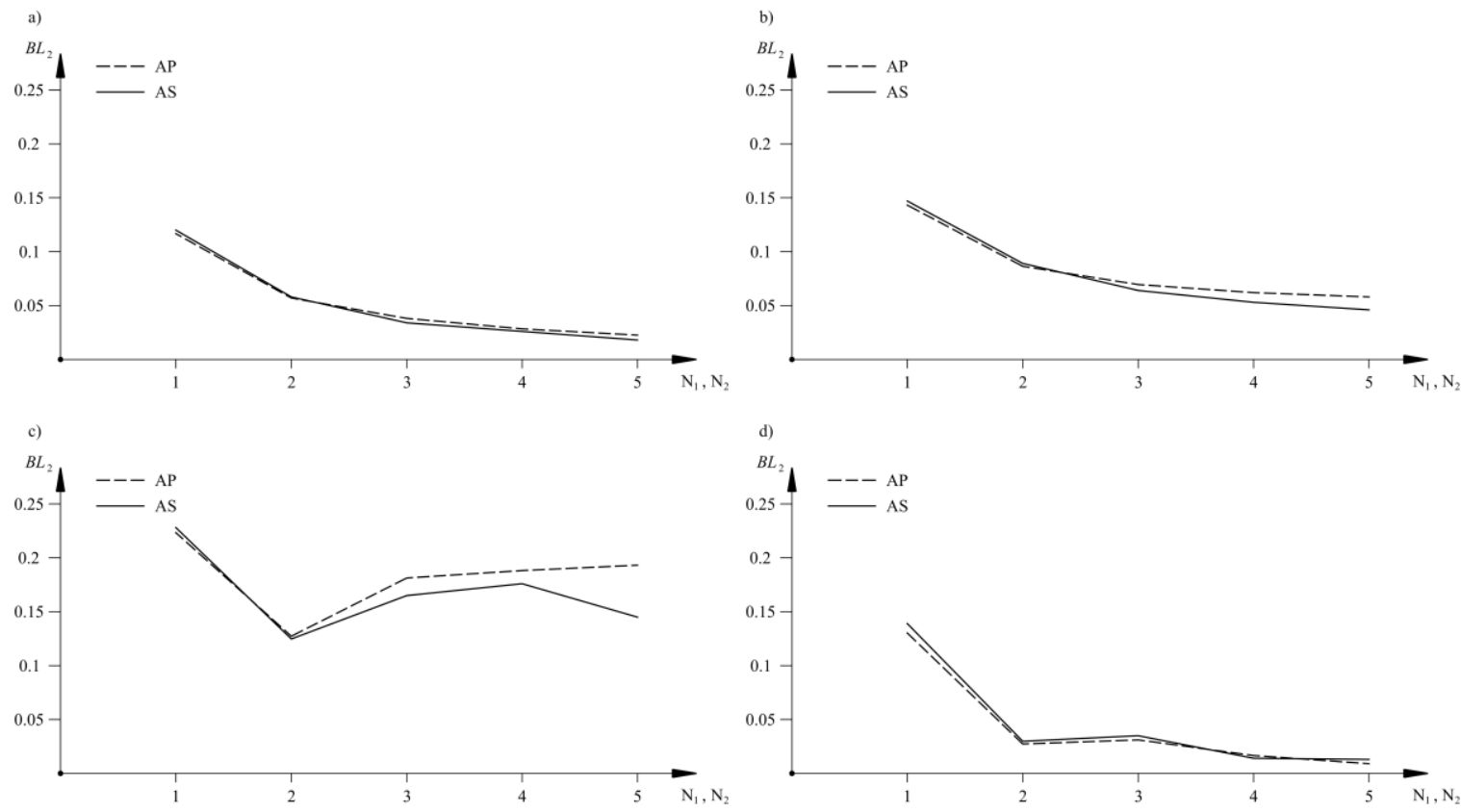

Fig. 10 Influence of capacity of buffers on the probability of blockage of the $2^{\text {nd }}$ machine for line a) $\mathrm{L}_{1}$, b) $\mathrm{L}_{2}$, c) $\left.\mathrm{L}_{3}, \mathrm{~d}\right) \mathrm{L}_{4}$
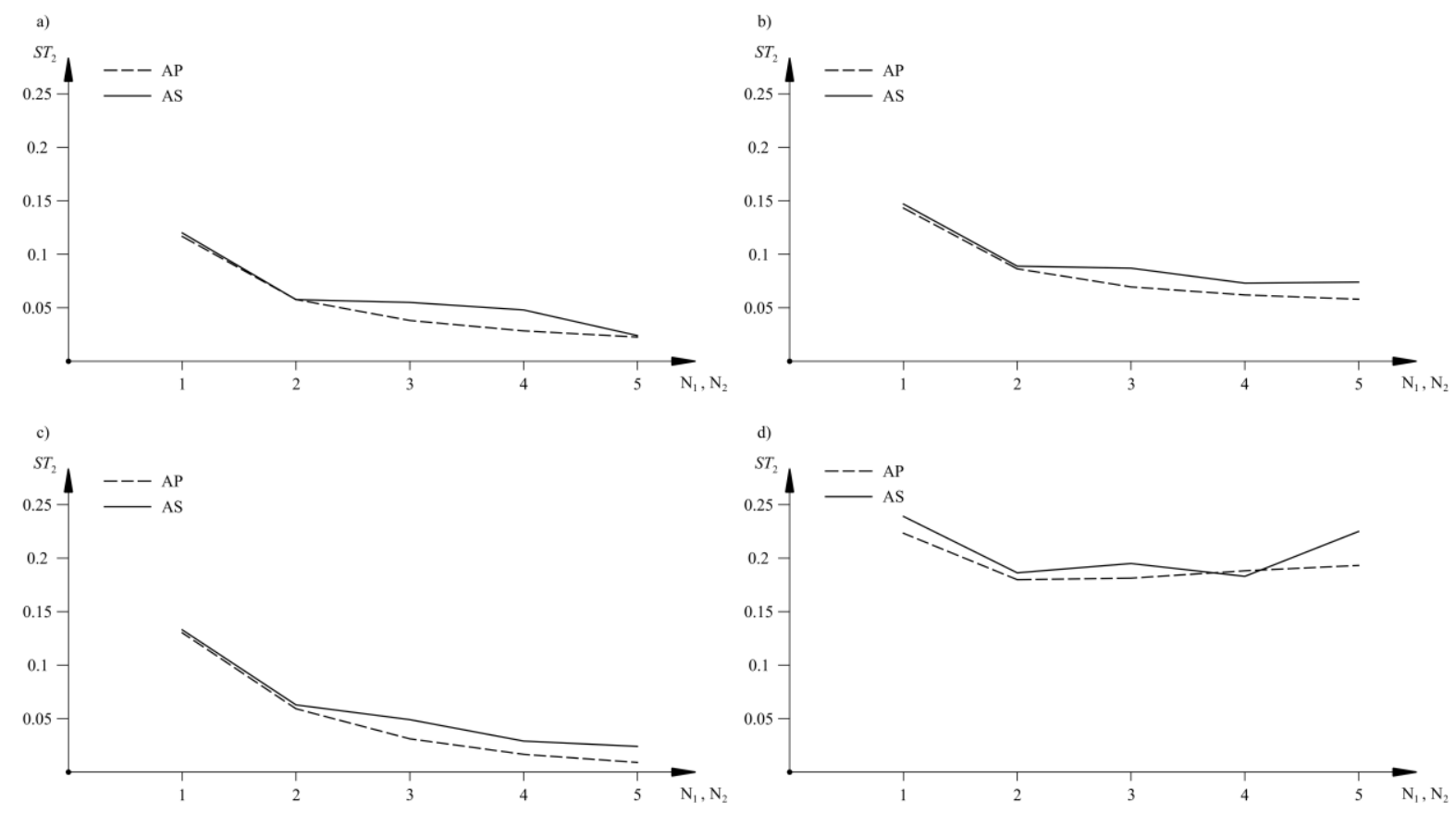

Fig. 11 Influence of capacity of buffers on the probability of starvation of the $2^{\text {nd }}$ machine for line a) $L_{1}$, b) $L_{2}$, c) $\mathrm{L}_{3}$, d) $\mathrm{L}_{4}$ 

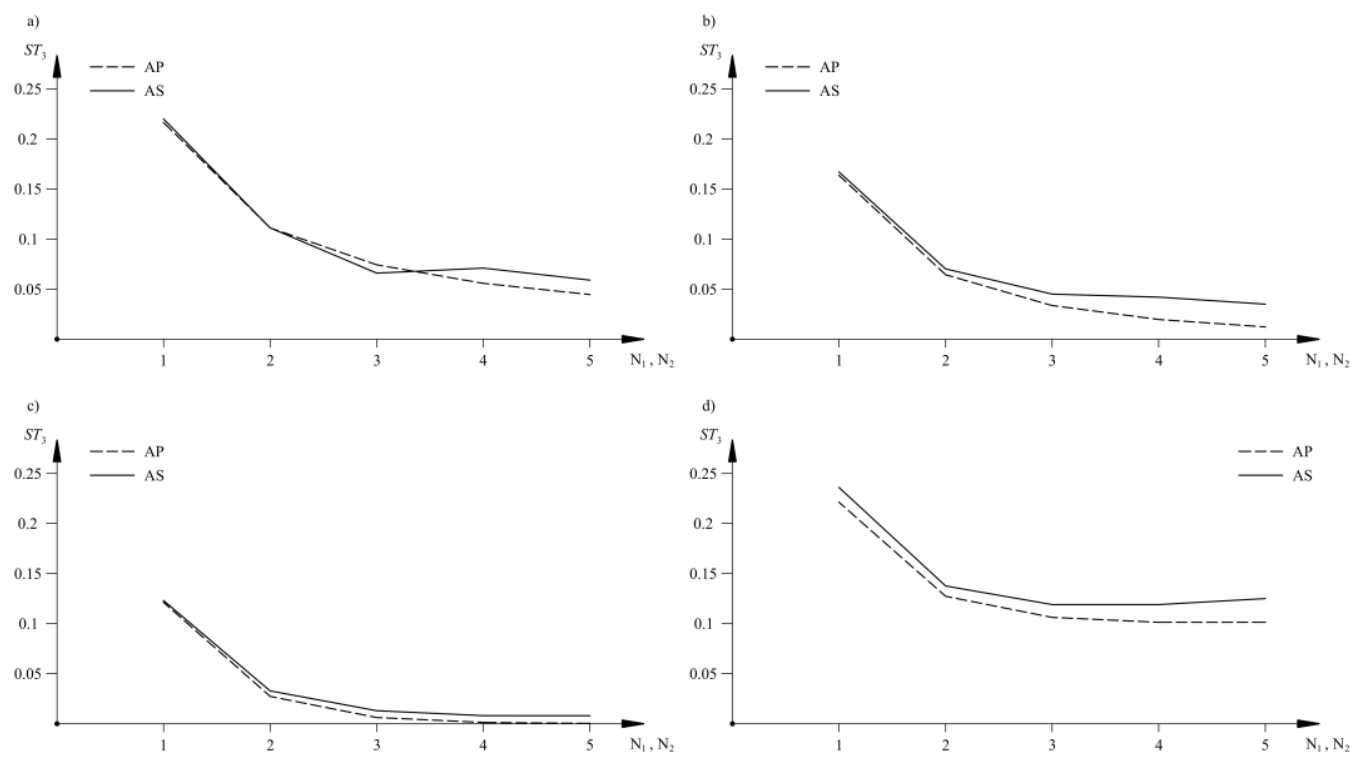

Fig. 12 Influence of capacity of buffers on the probability of starvation of the $3^{\text {rd }}$ machine for line a) $\mathrm{L}_{1}$, b) $\mathrm{L}_{2}$, c) $\left.\mathrm{L}_{3}, \mathrm{~d}\right) \mathrm{L}_{4}$

Comparison of influence of buffer capacity on production rate and work-in-process of the $1^{\text {st }}$ and the $2^{\text {nd }}$ machine shows good agreement between the results obtained applying analytical and approximated solution. Larger discrepancies can be noticed in cases of line $L_{3}$ $\left(W I P_{1}\right)$, Fig. 7 and lines $L_{2}$ and $L_{3}\left(W I P_{2}\right)$, Fig. 8, particularly for $N=5$. Global trend remains the same, and some useful asymptotic properties can be noticed, following [1]. When $N$ tends to infinity, production rate asymptotically approaches to the minimum of probabilities $p_{i}$. Also, work-in-process curves increase almost linearly with gradient depending on properties of machines. Lager discrepancies can be found in cases of blockage and starvation probabilities, Figs. $9-12$, however global behavior of curves remains the same except in cases of $L_{3}\left(B L_{1}\right)$, Fig. 9 and $L_{3}\left(B L_{2}\right)$, Fig. 10 where significantly lower probability in cases when $N=5$ can be found using analytical approach.

\section{Discussion}

Application of the presented research, as well as of the existing PSE research body within real manufacturing surrounding and particularly ship production process is a complex task. Different aspects of the problem have to be taken into account in order to address the problem effectively. The first question that is naturally imposed is related to modelling complexity. On one hand, a number of 3D simulation tools with amazing graphical representation of the processes are available at the market, however due to long term modelling requirements they may not be useful for production system design purposes. On the other hand, semi-analytical and analytical methods enable fast modelling and analysis with appreciable results, yet they involve rather complex theoretical background complicating their development. This point indicates critical necessity for further research on this topic, particularly regarding harsh industrial surroundings of the ship production process.

Reliability of modelling and input data is the second most important issue related to the production process analysis and mathematical modelling. In fact, there is no purpose of sophisticated analytical stochastic models development that would in turn use deterministic input data. It is therefore inevitable to invest further effort to investigate the nature of ship production surroundings, especially related to machine reliability models as well as properties 
of buffers. Even more importantly, to take into account probability and occupancy as functions of time leading towards dynamic or real life response of production lines.

Given such circumstances, the developed analytical solution of steady state behaviour of Bernoulli serial production line represents the first step towards general solution of the problem capable of realistic and simple production process modelling with output result that are easily interpreted and validated using measurements on shipyard floor.

\section{Conclusion}

Production system engineering, in order to fulfill its purpose, is strongly relied on mathematical models of systems under consideration. Three types of models were identified, i.e. analytical, semi-analytical and numerical, while particularly emphasizing the complexity of the model and its solution. Analytical solution of steady-state behavior of serial Bernoulli production line with three machines and two buffers is developed based on Markov chain approach and using eigenvalue and eigenvector analysis. Transition matrices in general case including three machines with different probabilities of failure and arbitrary occupancy of two buffers were formulated along with equations for performance measures, i.e. production rate, work-in-process and probabilities of blockage and failure. In such a way, analytical solution of the steady sate behavior of serial Bernoulli production line with three machines and two finite buffers is developed and presented for the first time.

The developed theory was illustrated using four different serial Bernoulli lines and the obtained results are compared to those determined using semi-analytical approach via aggregation procedure. Finally, the existing discrepancies are analyzed and pointed out for five different levels of buffers occupancy. From general perspective, good agreement has been found for most of the results within the examined space of system states with some exceptions related to probabilities of blockage and starvation that could influence bottleneck analysis. The developed analytical solution of steady state response of Bernoulli serial line with two buffers enabled further validation of aggregation procedure as to the present it was benchmarked only using extensive simulations.

As the developed theory is not restricted to considered cases, more general analytical solution of steady-state behavior of serial and assembly Bernoulli lines with arbitrary number of machines will be considered in further research. Along with that, transient behavior of Bernoulli lines can be investigated analytically in the same manner. In order to improve modelling reliability and enhance the applicability of the considered theory shipyard floor uncertainties should be taken into account in a sophisticated way.

\section{REFERENCES}

[1] Li, J. and Meerkov, S. M. (2009) Production System Engineering, Springer, New York. https://doi.org/10.1007/978-0-387-75579-3.

[2] Sevast'yanov, B. A. (1962) Influence of Storage Bi Capacity on the Average Standstill Time of Production Line. Theory of Probability Applications, pp. 429-438. https://doi.org/10.1137/1107040.

[3] Lim, J. T., Meerkov S. M. and Top, F. (1990) Homogenous, Asymptotically Reliable Serial Production Lines: Theory and Case Study. IEEE Transactions on automatic control, 35 (5), pp. 524-534. https://doi.org/10.1109/9.53518.

[4] Jacobs, D. and Meerkov, S. M. (1995) A system-theoretic property of serial production lines: improvability. International Journal of System Science, 26:4, pp. 755-785. https://doi.org/10.1080/00207729508929067

[5] Chiang, S. Y., Kuo, C. T. and Meerkov, S. M. (2000) DT bottlenecks in Serial Production Lines: Theory and Application. IEEE Transactions on robotics and automation, 16 (5), pp. 567-580. https://doi.org/10.1109/70.880806. 
[6] Meerkov, S. M. and Zhang, L. (2008) Transient behavior of serial production lines with Bernoulli machines. IIE Transactions 40, pp. 297-312. https://doi.org/10.1080/07408170701488037.

[7] Paul, S. and Ganguly S. (2013) Performance analysis of production lines with Bernoulli's machines. International Journal of Research in Engineering and Technology, 2 (12),.pp. 178-181. https://doi.org/10.15623/ijret.2013.0212032.

[8] Bergmann, S. and Strassburger, S. (2010) Challenges for the automatic generation of simulation models for production systems. Proceedings of the 2010 Summer Computer Simulation Conference, pp. 545-549.

[9] Hadjina, M., Fafandjel, N. and Matulja, T. (2015) Shipbuilding production process design methodology using computer simulation. Brodogradnja, 66 (2), pp. 77-91.

[10] Bronstein, I. N., Semendjajev, K. A., Musiol, G., and Muhlig, H. (2004) Mathematical handbook, Golden marketing, Zagreb. (in Croatian)

[11] Gershwin, S. B. and Schick, I. C. (1983) Modeling and Analysis of Three-Stage Transfer Lines with Unreliable Machines and Finite Buffers. Operations Research, 31 (2), pp. 354-280. ttps://doi.org/10.1287/opre.31.2.354.

[12] Dallery, Y. and Gershwin, S. B. (1992) Manufacturing flow line systems: A review of model and analytical results. Queuing Systems, 12, pp. 3-94. ttps://doi.org/10.1007/BF01158636.

[13] Chang, S. H. and Gershwin, S. B. (2010) Modeling and analysis of two unreliable batch machines with a finite buffer in between. IIE Transactions 42, pp. 405-421. https://doi.org/10.1080/07408170903228934.

[14] Gebennini, E. and Grassi, A. (2015) Discrete-time model for two-machine one-buffer transfer lines with buffer bypass and two capacity levels. IIE Transactions 47, pp. 715-727.

https://doi.org/10.1080/0740817X.2014.952849.

[15] Pauše, Ž. (1985) Probability, Information, Stochastic processes, Školska knjiga, Zagreb. (in Croatian)

[16] Gantmacher, F. R. (1959) The theory of matrices, Chelsea Publishing Company, New York.

[17] Thompson, W. T. (1983) Theory of vibration with applications, George Allen \& Unwin, London.

[18] www.productionsystemengineering.com. Accessed at September 1, 2016.

\footnotetext{
Submitted: $\quad$ 5.09.2106. Hadžić Neven, neven.hadzic@ fsb.hr

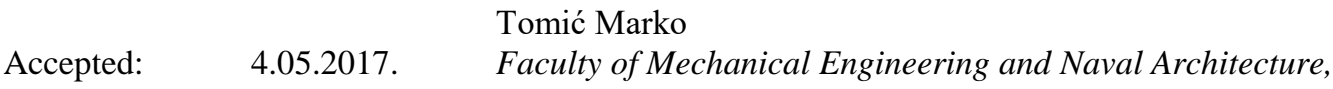
University of Zagreb, I. Lučića 5, 10000 Zagreb
} 